\title{
LA COERENZA TESTUALE DAL PUNTO DI VISTA TRADUTTIVO
}

Lo scopo del presènte contributo è presentare sinteticamente i risultati di una ricerca recente, volta ad indagare il fenomeno della coerenza come categoria testuale principale in chiave traduttiva. Dopo una discussione teorica del concetto in questione, l'autrice analizza le traduzioni slovene del Principe di Niccolò Machiavelli, che per la loro divergenza funzionale si rivelano particolarmente adatte allo studio dei meccanismi della coerenza testuale in prospettiva traduttiva.

Keywords: coerenza testuale, traduzione, funzionalismo, contesto, II Principe machiavelliano

\section{Introduzione}

Lo studio della coerenza testuale in testi tradotti ${ }^{1}$ è stato motivato dalla seguente osservazione: rispetto ai loro originali le traduzioni risultano spesso caratterizzate da una specie particolare di modificazioni che, tipicamente, si rivelano come aggiunte di vari elementi linguistici o di gruppi di tali elementi. Si tratta non di addizioni causate da costrizioni grammaticali, del resto operanti a vari livelli linguistici, da quello sintagmatico a quello testuale, ${ }^{2}$ bensì di inserzioni puramente funzionali, dettate quindi dal ruolo che il testo tradotto presumibilmente svolgerà nella cultura d'arrivo. Le modificazioni in questione non sono quindi motivate dal sistema della lingua d'arrivo e non rappresentano casi di espansioni morfo-sintattiche dovute alla complessità dell'originale. D'altra parte, non si tratta neppure di modifiche simili a quelle che si possono riscontrare in traduzioni eseguite da traduttori semi-professionali o dagli studenti, che - com'è noto - tendono a usare delle soluzioni parafrastiche, spesso infondate e soggettive, che possono essere dovute sia a difficoltà linguistiche sia a negligenza (v. Ožbot 1997). ${ }^{3}$

1 Su questo studio si basa la tesi di dottorato dell'autrice discussa il 12 maggio 2000 all'Università di Ljubljana (relatrice: prof.ssa Tjaša Miklič). V. Ožbot (1999).

2 I seguenti due sono esempi di modificazioni sistemiche dovute a costrizioni grammaticali della lingua d'arrivo, cioè lo sloveno, rispetto all'italiano come lingua di partenza. Nel primo caso si tratta di modificazione sistemica al livello di sintagma nominale e nel secondo al livello frastico:

a. Ha ottenuto il massimo. vs Dobil je najvišjo oceno.

Per esprimere il concetto del »massimo dei voti« in sloveno non si può usare il superlativo sostantivato, tipico in italiano, bensì si ricorre, normalmente, a un sintagma nominale consistente del nome »ocena ("voto«) al quale viene preposto il superlativo dell'aggettivo »visok« (»alto《) o sim.

b. É andata da Clara per aiutarla. vs Šla je h Klari, da bi ji pomagala.

Diversamente da quanto si può osservare nelle dipendenti finali (ed alcune altre) italiane, in sloveno l'identità tra il soggetto della dipendente e della principale non determina la scelta della forma verbale indefinita nella dipendente; nelle finali slovene, anche nei casi del genere viene usata la forma finita.

Uno studio dettagliato di espansioni morfo-sintattiche del genere riscontrabili in traduzioni slovene di testi inglesi offre Milojević Sheppard (1993).

3 Un caso di tale espansione infondata si può osservare nel seguente passo tratto dalla traduzione slovena (Zgodovina italijanske književnosti, Ljubljana: Državna založba Slovenije, 1967; traduttore: Bogomil Fatur) 
Le modificazioni alle quali sarà rivolta la nostra attenzione sono di natura diversa. Data la loro motivazione funzionale, esse si possono considerare come elementi che servono a rafforzare la coerenza del testo d'arrivo, cioè quella proprietà di esso in base alla quale il destinatario sarà in grado di costruire il senso del testo. Si tratta dunque di elementi introdotti dal traduttore per aumentare la trasparenza delle relazioni del mondo testuale e, conseguentemente, per facilitare il processo della comprensione del testo. Provvisoriamente tali elementi si potrebbero chiamare modificatori funzionali. Essendo questi modificatori condizionati da fattori contestuali ${ }^{4}$ non ci deve sorprendere che traduzioni di uno stesso testo per le quali sono previste funzioni distinte si differenzieranno anche per il fatto che conterranno diversi modificatori funzionali, appunto in dipendenza dai diversi ruoli che sono destinati a svolgere.

Prima di esaminare materiale testuale concreto sarà opportuno definire con più esattezza il concetto di coerenza testuale e discutere la sua pertinenza nell'ambito traduttivo.

\section{Coerenza testuale: alcuni tratti fondamentali ${ }^{5}$}

Nell'ambito della linguistica testuale e in genere in quello della linguistica funzionale è generalmente riconosciuto che la coerenza sia una caratteristica testuale fondamentale, dato che è in base ad essa che possiamo distinguere i testi come unità comunicative di base dai non-testi, cioè cumuli di materiale linguistico che non sono in grado di svolgere la funzione comunicativa. Pur essendo stata indagata in sedi diverse (per es., nel campo della linguistica testuale ed dell'analisi del discorso, della linguistica applicata, della pragmatica, della psicolinguistica, della linguistica cognitiva, della neurolinguistica e, in parte, anche nel campo della teoria della traduzione $)^{6}$, la coerenza testuale rimane un fenomeno per molti lati sconosciuto, dato che i meccanismi del suo funzionamento non sono ancora stati spiegati in maniera soddisfacente. Ciò sarà in buona parte dovuto al fatto che i meccanismi cognitivi che regolano la costituzione del

di Storia della letteratura italiana di Attilio Momigliano (Milano/Messina: Giuseppe Principato, 1959). Nel brano viene presentato lo scritto manzoniano Del romanzo e in genere de 'componimenti misti di storia e d'invenzione, nel quale il grande scrittore è critico del genere letterario in questione:

- Il valore del discorso è quindi da ricercarsi non in questa condanna, smentita dai fatti, ma nello studio dell'evoluzione del poema epico e della tragedia storica alla luce dell'idea che il progresso della storia viene trasformando il campo dell'arte. (p. 458)

- Vrednosti razprave torej ne smemo iskati v sami obsodbi, kateri so tehtnost jemala dejstva, ampak v preučevanju razvoja epske pesnitve in zgodovinske tragedije v luči ideje, da zgodovinski napredek spreminja tudi področje in oblike umetnosti. (p. 455)

Il sintagma sloveno »področje in oblike umetnosti« si riferisce al »campo ed alle forme dell'arte«. Siccome l'originale parla esclusivamente del »campo dell'arte« come ambito generico di un'attività umana, l'introduzione del lesema »oblike« (»forme«) non è altro che fuorviante.

4 Contesto viene inteso come l'insieme dell'ambiente linguistico ed extralinguistico del testo.

5 Per una presentazione più estesa di quanto viene esposto nei capitoli Il e III del presente articolo v. Ožbot (ics).

6 Sui contributi allo studio della coerenza fatti da esponenti di vari approcci v. Viehweger (1989), van de Velde (1992), Hellman (1995). Rassegne bibliografiche sono offerte in Petöfi (cur.) (1988: 478-501), in Heydrich et al. (cur.) (1989: 383-396) e in Bublitz et al (cur.) (1999: 267-295). Sulla coerenza testuale nell'ambito della teoria della traduzione v. Reiß/Vermeer (1984) e Resch (1997); cfr. anche capitolo III del presente articolo. 
senso nel processo di interpretazione e di produzione testuale non ci sono ancora noti, nonché al fatto che il funzionamento dei testi in situazioni concrete sia troppo complesso e venga determinato da troppi parametri, sia linguistici che extralinguistici, perché possa essere studiato a fondo con gli strumenti analitici di cui attualmente disponiamo.

Tenendo presenti questi limiti, possiamo tuttavia individuare alcune caratteristiche fondamentali della coerenza testuale indispensabili come base per indagini successive di questo fenomento testuale:

1. La coerenza testuale è la caratteristica fondamentale di tutti i testi, in quanto unità di materiale linguistico dotate di senso, e va considerata come la conditio sine qua non per la costituzione del senso del testo. Essa è quindi la categoria centrale dalla quale dipende la stessa testualità dei testi, cioè la caratteristica per la quale essi possono svolgere il loro ruolo di unità comunicative principali.

2. La costituzione della coerenza testuale può avvenire solamente tramite il ricevente, che nel processo di interpretazione del testo cerca di costruirne il senso. Perché in questo processo la costruzione del senso possa avverarsi, è prima necessario che l'autore del testo tenga conto del previsto contesto situazionale, che rispetti, almeno fino a un certo grado, le norme grammaticali e le convenzioni testuali $\mathrm{di}$ una data lingua e che presenti il mondo testuale in modo tale che il ricevente sarà in grado di interpretarlo.

3. Perché il testo possa essere considerato coerente, non è necessario che queste condizioni siano soddisfatte al massimo. Anche se un testo viene percepito come localmente incoerente, ciò non significa che la sua coerenza globale non potrà essere costituita. Com'è ben noto, avviene piuttosto raramente che il ricevente trovi incoerente un testo (cfr. Halliday/Hasan 1976: 24), anche quando esso si presenta difettoso sotto vari aspetti (ad es. dal punto di vista grammaticale o dal punto di vista della distribuzione delle informazioni). Questo non deve sorprenderci, dato che la coerenza testuale non è altro che una sottospecie della »coerenza generale«, che sempre cerchiamo di costruire nelle situazioni in cui ci troviamo, anche se per interpretarle non sempre abbiamo a che fare con del materiale linguistico.

4. Nel processo di interpretazione del testo, la coerenza normalmente non viene ricercata dal ricevente in maniera consapevole, eccetto nei casi in cui questo processo non produca risultati accettabili. Pertanto la coerenza testuale può giustamente essere considerata un »epifenomeno«, come suggerisce Christina Hellman (1995: 190-191).

5. Se un testo è considerato coerente, questo non significa che tutte le relazioni del mondo testuale vi siano espresse esplicitamente. Il grado di coerenza non dipende direttamente dal numero e/o dalla densità di relazioni esplicitate. Per interpretare qualsiasi testo è necessario che il ricevente attivi la propria capacità inferenziale. Per il produttore del testo sarà invece importante saper decidere, in base alla funzione del testo prevista, quali informazioni esprimere esplicitamente e quali in maniera implicita - nella misura in cui questo dipenda realmente dal produttore e non sia determinato dallo stesso sistema della lingua del testo (ad es. l'uso del duale in sloveno). 
6. Per poter interpretare un testo, l'enciclopedia mentale del ricevente deve esere abbastanza grande e tra il produttore ed il ricevente deve esistere un sapere comune, che comprende la conoscenza del mondo, la conoscenza della realtà presentata nel testo, la conoscenza di generi e convenzioni testuali, ecc.

Da quanto è stato finora constatato consegue che per la costituzione della coerenza testuale è necessario che siano soddisfatte due condizioni fondamentali, che pertanto possiamo chiamare condizioni di coerenza: da una parte deve esserci il materiale testuale come base oggettiva che potrà essere interpretata dal ricevente e, dall'altra, quest'ultimo deve avere la capacità di interpretarlo realmente, il che necessariamente presuppone anche l'elemento soggettivo. La coerenza viene quindi costituita quando il ricevente riconosce le relazioni del mondo testuale, ovvero la »continuità del senso« del testo (Beaugrande/Dressler 1992: 65).

Dato che la costituzione della coerenza coinvolge in parte anche la soggettività del ricevente, è facile capire che lo stesso testo può risultare coerente per certi riceventi e incoerente per gli altri. Talvolta le discrepanze nella percezione della coerenza possono essere legate a differenze interculturali ossia a differenze dei saperi tipicamente posseduti da membri di culture diverse. Da quest'ottica, la coerenza testuale si rivela come un concetto che concerne in modo particolare il processo della traduzione. Il fatto sta che nei testi d'arrivo che rappresentano traduzioni di testi di partenza coerenti la costituzione della coerenza non è resa possibile automaticamente, bensì spesso presuppone che il traduttore produca il testo tenendo conto delle caratteristiche specifiche della cultura pertinenti alla interpretazione del testo in questione.

\section{La traduzione come processo di produzione testuale}

Secondo la teoria della traduzione di stampo funzionale, e soprattutto secondo la Skopostheorie di Hans J. Vermeer e Katharina Reiß (v. Reiß/Vermeer 1991: 109-118), il processo traduttivo ha come scopo la produzione di un testo d'arrivo che si basi sul testo di partenza e che dovrebbe essere in grado di svolgere la funzione comunicativa prevista nella cultura d'arrivo. Anche se le funzioni del testo di partenza e del testo d'arrivo saranno uguali - ad es. anche se una guida turistica come testo di partenza svolgerà la stessa funzione pure quando farà parte, come traduzione, della cultura d'arrivo - il traduttore deve normalmente tenere conto di eventuali differenze nelle conoscenze culturali dei lettori d'arrivo rispetto a quelle dei lettori di partenza.

In base a considerazioni del genere Reiß and Vermeer propongono la distinzione tra la coerenza intratestuale e la coerenza intertestuale. La prima si riferisce alla coerenza del testo di partenza e del testo d'arrivo come due unità comunicative separate, ovvero funzionalmente indipendenti; la seconda, invece, riguarda la relazione tra il testo di partenza ed il testo d'arrivo e può anche essere designata come »fedeltà«. In prospettiva funzionale, è ovvio che risulterà più importante la coerenza intratestuale, appunto perché il processo di traduzione sarà sempre soprattutto un processo di pro- 
duzione testuale per il pubblico d'arrivo, che normalmente non è in grado di utilizzare il testo di partenza, del quale la traduzione di solito rappresenta una sostituzione funzionale. Pertanto il legame che la traduzione ha con l'originale risulta meno importante del suo potenziale funzionale nella cultura d'arrivo. Dato ciò, è naturale che la traduzione vada vista come un'operazione molto diversa dalla semplice sostituzione di unità del testo di partenza con delle unità del testo d'arrivo.

Osserviamo ora i meccanismi testuali della coerenza in base all'analisi di traduzioni slovene del Principe di Niccolò Machiavelli.

\section{La coerenza testuale nelle traduzioni slovene del Principe di Machiavelli}

La coerenza testuale in testi tradotti è stata studiata in traduzioni slovene di vari testi machiavelliani tra i quali si è rivelato particolarmente interessante Il principe (1513), siccome esiste in più traduzioni alle quali sono state assegnate, a quanto pare, funzioni assai diverse. La prima versione slovena del Principe risale al 1920, quando lo tradusse Albin Prepeluh (1880-1938), un uomo politico di tendenze social-democratiche, che lo pubblicò con lo pseudonimo significativo Abditus. La traduzione successiva apparve nel 1966 ed è, come quella più recente (1990), opera di Niko Košir (1919-2000), scrittore, professore di letteratura italiana e, soprattutto, traduttore di rilievo. ${ }^{7}$ Le due traduzioni di Košir sono quasi identiche, dato che la seconda rappresenta una versione riveduta e linguisticamente aggiornata della prima. ${ }^{8}$ Esse presentano però differenze sensibili rispetto alla versione di Prepeluh, il che sembra dovuto al fatto che i due traduttori abbiano destinato i loro testi a funzioni distinte.

\section{IV.1 Il principe in sloveno: alcune osservazioni preliminari}

Le differenze funzionali tra la versione di Prepeluh e quella di Košir derivano dalla stessa polivanenza del testo originale, che permette sia una lettura in chiave pragmatica, come saggio storico e politico, sia una lettura in chiave piuttosto sovrapragmatica, come testo semiletterario (cfr. Vipond/Hunt 1989: 157). Albin Prepeluh mise in rilievo soprattutto il lato pragmatico dell'opera machiavelliana, che cercò di presentare al lettore sloveno come saggio analitico con valore prevalentemente storico-politico; Niko Košir, invece, tenne conto tanto degli aspetti contenutistici quanto di quelli espressivi, riuscendo così a produrre un testo, che per la sua perfezione stilistica e la tendenza verso il letterario, supera il livello puramente pragmatico.

Che la traduzione di Košir sia destinata a scopi non immediatamente pragmatici viene forse indicato anche dal fatto che la versione del 1966 fa parte del »Sivi kondor«,

7 Tradusse, tra l'altro, vari classici della letteratura spagnola, italiana e francese (per es. Poema de mio Cid, Don Chisciotte di Cervantes e La vida es sueño di Calderon; Il Decameron di Boccaccio, Il fu Mattia Pascal di Pirandello; La princesse de Clèves di Mme de la Fayette ecc.), nonché opere filosofiche (per es es. Del sentimiento trágico de la vida di Miguel de Unamuno) e trattati di storia dell'arte (per es. Dietro l'immagine di Federico Zeri).

8 Dato che le differenze tra le due versioni koširiane sono minime e non hanno alcuna importanza per gli aspetti testuali esaminati in questo articolo, non le tratto separatamnente. 
una collana che comprende prevalentemente opere letterarie, e che quella del 1990 sia inserita nella »Filozofska knjižnica«, letteralmente »Biblioteca filosofica«, cioè una collana di cui fanno parte soprattutto i classici di filosofia. In base alla supposta diversità funzionale delle due traduzioni si può anche prevedere che siano destinate a due gruppi distinti di lettori: Prepeluh sembra aver avuto in mente un pubblico interessato soprattutto al messaggio storico e/o politico del Principe, mentre la traduzione di Košir sembra poter soddisfare anche il lettore che abbia interessi sovrapragmatici.

La divergenza funzionale tra le traduzioni di Prepeluh e di Košir dimostra che la necessità di produrre traduzioni nuove di testi già tradotti in una data cultura non sorge solo in situazioni in cui le traduzioni precedenti vengono percepite come inaccurate o antiquate dal punto di vista linguistico (anche se nella traduzione di Prepeluh si potrebbe, in realtà, parlare anche di problemi del genere). Tale necessità viene, in realtà, percepita anche nei casi in cui un testo offre possibilità di lettura nuove dal punto di vista funzionale. Vediamo ora la eterogeneità funzionale delle traduzioni slovene in maniera più dettagliata.

\section{IV.1.1 La traduzione di Albin Prepeluh}

Albin Prepeluh tentò di offrire l'opera machiavelliana al lettore sloveno come saggio politico attuale. La traduzione fu pubblicata dopo il Primo conflitto mondiale, il traduttore se ne era però occupato già negli anni di guerra, come precisa alla fine dell'Introduzione (v. p. 16). Quest'introduzione, pur avendo come scopo principale la presentazione dei tempi, della vita e delle opere di Machiavelli, nonché delle circostanze in cui Il principe venne scritto, si conclude in spirito molto attualistico. Prepeluh mette in risalto la modernità del pensiero machiavelliano, cercando di trarre dei paragoni tra l'Italia ai tempi di Machiavelli e la realtà slovena in cui viveva il traduttore: »Cosa vogliamo da Machiavelli e dalle sue dottrine noi sloveni? /.../ Quando Machiavelli scriveva 'Il principe' la sua patria era smembrata, umiliata, distrutta, saccheggiata. Anche la nostra si trova in uno stato simile $/ \ldots / \ll^{9}$ (ibid.; trad. M.O.). Prepeluh sottolinea anche la strumentalità del Principe come testo dal quale il lettore moderno possa imparare molto per poter un giomo celebrare »la vittoria«, della quale non dice nulla di preciso.

Alla luce dello scopo fondamentalmente pragmatico di questa traduzione, vanno spiegate anche numerose caratteristiche del testo che servono ad avvicinare al lettore il messaggio del testo e a facilitarne la comprensione. ${ }^{10}$ Ad esempio, i titoli dei singoli capitoli non sono in latino, come nell'originale, bensì in sloveno; ${ }^{11}$ le citazioni latine sono corredate di una traduzione slovena a piè di pagina. Si trovano in calce anche altre note, del resto non numerosissime, che sono di natura esplicativa e si riferiscono soprattutto a personaggi, luoghi e fatti del mondo testuale, oppure ad altre opere di

\footnotetext{
9 »Kaj hočemo $z$ Machiavelijem in njegovimi nauki mi Slovenci? /.../Ko je Machiavelli ustvarjal 'Vladarja' je bila njegova domovina razkosana, ponižana, razbita, oplenjena. Tudi naša domovina je danes $v$ podobnem stanju $/ \ldots / . k$ Circa i cosiddetti »spostamenti di coerenza《 (»shifts in coherence《), condizionati dall'intenzione del traduttore di avvicinare il mondo testuale al pubblico d'arrivo previsto, v. Blum-Kulka (1986: 23-29).

11

Lo stesso vale anche della più recente delle due traduzioni di Košir.
} 
Machiavelli e alle sue posizioni storico-politiche.

Anche dal punto di vista prettamente linguistico la traduzione di Prepeluh risulta pragmatica: l'intenzione del traduttore è soprattutto trasmettere al ricevente l'essenziale del messaggio. Pertanto è naturale che, nei confronti dell'originale, sia piuttosto libera - e in alcuni luoghi anche sbagliata per motivi di scarse competenze professionali del traduttore - e che il traduttore non sembri badare molto al lato espressivo, il che, come appare chiaro dal esempio seguente, lo rende molto diverso da Košir:

(1)

* E questo è che alcuno è tenuto liberale, alcuno misero /.../; alcuno è tenuto donatore, alcuno rapace; alcuno crudele, alcuno pietoso; l'uno fedifrago, l'altro fedele; l'uno effeminato e pusillanime, l'altro feroce e animoso; l'uno umano, l'altro superbo; l'uno lascivo, l'altro casto; l'uno intero, l'altro astuto; l'uno duro, l'altro facile; l'uno grave, l'altro leggieri; l'uno religioso, l'altro incredulo, e simili. (P 90-91) ${ }^{12}$

* Se pravi, da imajo nekoga za darežljivega, drugega za stiskača /.../; tega imajo za radodarneža, onega za grabežljivca, tega za okrutneža, onega za milosrčnega, ta jim je verolomnež, oni mož beseda; ta mehkužnež in bojazljivec, oni krut in pogumen, ta človeški, oni prevzeten, ta pohoten, oni zdržen, ta ves scela, oni lisjak, ta neupogljiv, oni lahkomiseln, ta resen, oni frfra, ta veren, oni neveren, in tako dalje. (K 57)

* Zato imenujemo tega darežljivca, onega pa skopuha /.../; enega radodarneža, drugega grabljivca; enega okrutneža, drugega usmiljenega; enega verolomneža, drugega zvestega; enega mehkužnega in malodušnega, drugega neupogljivega in srčnega; enega človekoljubnega, drugega ošabnega; enega pohotnega, drugega zdržljivega; tega odkritega, onega zvitega; tega trdosrčnega, onega lahkomiselnega; tega resnega, onega površnega; tega vernega, drugega nevernega in tako dalje. (Prep 70)

Osservando i lessemi messi in rilievo è facile vedere che, rispetto a Košir, Prepeluh sia molto meno attento all'aspetto espressivo. I lessemi da lui usati sono neutri, mentre quelli nel testo koširiano risultano stilisticamente marcati. Da uomo politico, Prepeluh cerca soprattutto di trasmettere al lettore d'arrivo informazioni sui fatti, l'esseziale del pensiero politico di Machiavelli, mentre per Košir alla completezza del messaggio sembra contribuire tanto l'informazione quanto il modo di presentarla. ${ }^{13}$ Così il passo di Košir è contraddistinto da una combinazione di lessemi che rimanda allo stesso tempo al registro letterario e all'arcaico (ad es. il lessema »milosrčen« per

Le maiuscole tra parentesi designano i testi esaminati: P - Il Principe

Prep - Il Principe nella traduzione di Prepeluh

I numeri indicano le pagine dove si trovano i brani discussi.

K - Il Principe nella traduzione di Košir (1990).

13 È interessante che la traduzione di Košir del 1966 si avvicini di più alla versione di Prepeluh che non la traduzione koširiana posteriore. Come si può dedurre dall'analisi comparativa delle versioni dei due traduttori, Košir, almeno nella preparazione della prima versione, sembra aver consultato il testo di Prepeluh, data però la sua tendenza a una perfezione stilistica sempre più grande, gli echi del testo di Prepeluh, nella versione più recente, si fanno meno sentire. 
»pietoso《), nonché all'idiolettale (ad es. il sostantivo »frfra« per l'espressione machiavelliana »leggieri《). In questo modo il traduttore presenta una versione piuttosto distaccata del mondo testuale nella quale le scelte stilistiche fanno parte integrante del messaggio.

\section{IV.1.2 La traduzione di Niko Košir}

Se l'interesse per l'opera di Machiavelli nutrito da A. Prepeluh può essere caratterizzato come interesse »politico«, l'approccio di Košir è essenzialmente filologico. Suo scopo fu quello di presentare al lettore sloveno un Machiavelli piuttosto letterarizzato, per cui la traduzione di Košir è in grado di soddisfare tanto i lettori che si interessano di letteratura italiana quanto quelli interessati alla storia politica. Tuttavia le intenzioni di Košir non sono divulgative: diversamente da Prepeluh, Košir non offre il testo macchiavelliano come mezzo di educazione o di istruzione politica, e pertanto non cerca a tutti costi di avvicinare il testo al lettore. Se l'atteggiamento di Prepeluh nei confronti del testo machiavelliano era relativamente personale, Košir si presenta più distaccato. Il suo testo è destinato soprattutto a un pubblico colto, che lo userà sia come testo letterario, sia come classico storico-politico, meno probabilmente come testo informativo immediatamente pertinente all'azione politica. Nella versione del 1990 tale orientamento viene confermato anche dallo studio introduttivo di Atilij Rakar, che offre una presentazione approfondita della vita, delle opere e dei tempi di Machiavelli. Nella versione del 1966, invece, l'introduzione, scritta da Vlado Vodopivec, si concentra di più su aspetti politici dell'opera di Machiavelli e, come quella di Prepeluh, tende ad attualizzarla, talvolta anche tramite riferimenti ad alcuni pensatori socialisti come Engels e Gramsci; siamo alla metà degli anni sessanta, quando poteva sembrare più prudente corredare un classico di storia politica italiana con qualche elemento di attualizzazione.

L'impiego di arcaismi e di elementi lessicali e strutture morfosintattiche tipiche della lingua letteraria, nonché il fatto che la maggioranza delle note - che sono dettagliate ed esaustive, e di tipo filologico, intertestuale e contenutistico - appaiano dietro il testo ci dimostrano che lo scopo di Košir non fu quello di produrre un testo che sarebbe potuto servire a un pubblico numeroso ed eterogeneo. Il traduttore partì da una supposizione più realistica: che gli scritti di Machiavelli non avrebbero attirato un pubblico vasto, ma piuttosto un pubblico specialistico, abituato a letture più esigenti e disposto a sforzarsi per poter arrivare al senso del testo.

Malgrado le differenze che intercorrono tra la traduzione di Prepeluh da una parte e le versioni di Košir dall'altra, tutti e tre i testi si dimostrano coerenti. I due traduttori furono ben consapevoli delle funzioni assegnate ai testi sloveni e fu sempre lo scopo previsto a determinare le loro strategie traduttive.

Nella parte seguente, cercheremo di esaminare i procedimenti tramite i quali la costituzione della coerenza testuale è resa possibile in traduzioni diverse come varianti eterofunzionali di uno stesso testo. 


\section{IV.2 I procedimenti relativi alla coerenza testuale nelle traduzioni slovene del Principe}

Ambedue i traduttori sloveni del Principe cercano di rendere possibile ai loro lettori la costruzione della coerenza testuale in base alla quale potranno riconoscere il senso del testo. A questo scopo vengono impiegati alcuni procedimenti volti a rendere il messaggio più esplicito e/o a facilitare il processo della elaborazione mentale del testo. Tali meccanismi testuali sono motivati esclusivamente dalla funzione prevista per il testo d'arrivo e non vengono introdotti per costrizioni sistemiche. Che le diverse traduzioni di uno stesso testo presentino delle differenze relative a tali procedimenti di coerenza testuale non è sorprendente; dato che la coerenza testuale è, almeno in parte, una categoria soggettiva, è naturale che la sua costituzione in un testo tradotto sarà, almeno in parte, filtrata attraverso l'interpretazione del traduttore.

I procedimenti volti a rendere possibile la costituzione della coerenza testuale sono i seguenti:

A. esplicazione,

B. aggiunta di espressioni semanticamente vicine,

C. introduzione di strutture parallele,

D. creazione di nuovi rapporti interfrastici,

E. uso di connettori testuali.

Osserviamo alcuni esempi di tali procedimenti più da vicino.

\section{IV.2.1 Esplicazione}

Il meccanismo più frequente e più tipico tramite il quale il senso del testo diventa più trasparente è l'esplicazione, ovvero la spiegazione, sia nel testo sia nelle note, di alcuni elementi del messaggio che nell'originale sono resi in maniera meno esplicita e che sono fondamentali per la comprensione del testo o di un dato brano del testo. Nel Principe tali elementi si riferiscono soprattutto a diverse entità del mondo testuale (ad es. a personaggi, luoghi fisici, fatti della storia ecc.); se non sono resi espliciti, la comprensione del testo può diventare difficile per la maggior parte dei lettori le cui conoscenze del mondo testuale non siano abbastanza specifiche per garantire una comprensione immediata.

Dell'esplicazione come meccanismo di coerenza si servono tutti e due i traduttori, il che è naturale, dato che la realtà storica presentata nel testo di partenza potrà apparire relativamente lontana tanto al pubblico di Prepeluh quanto a quello di Košir. Ecco un esempio tipico di esplicazione:

(2)

* In modo che, rimasa sanza vita, aspetta qual possa essere quello che sani le sue ferite, e ponga fine a' sacchi di Lombardia, alle taglie del Reame e di Toscana, e la guarisca di quelle sue piaghe già per lungo tempo infistolite. (P 134)

* Tako Italija še vedno kakor mrtva čaka, kdo bi utegnil biti tisti, ki bo zacelil njene rane, naredil konec plenitvam $v$ Lombardiji, vojnim dajatvam $v$ Neapeljskem kraljestvu in v Toskani ter ozdravil njene že dolgo prisajene tvore. (K 92) 
* Italija je še vedno kakor mrtva in čaka na nekoga, ki bi jo po hudih udarcih zopet ozdravil, napravil konec plenitbam $v$ Lombardiji, izžemanju in izsiljevanju v rimski državi, neapoljskem kraljestvu in Toskani in zlečil njene že ognojene rane. (Prep 100)

Nella traduzione del passo machiavelliano in cui il sostantivo »Reame « si riferisce al Regno di Napoli, ambedue i traduttori rendono il riferimento esplicito tramite l'aggiunta dell'aggettivo »neapeljski/neapoljski« davanti al nome »kraljestvo« (»regno«).

Diversamente da Prepeluh, che, privilegiando oltrettutto la concisione, si limita normalmente a rendere espliciti solo quegli elementi che sono centrali dal punto di vista della comprensione del messaggio, Košir tende ad aumentare la trasparenza del testo anche quando per arrivare al messaggio non è necessaria una conoscenza molto dettagliata della realtà extralinguistica, bensì solo una lettura attenta. Ad esempio, nel passo seguente, Košir usa la coppia di espressioni »vojaki«/《vojska« e »ljudstvo《 due volte (una delle quali come apposizione del lessema »sloj«/«ordine«/ al duale), mentre nell'originale appaiono la prima volta i lessemi »soldati« e »populi« e la seconda, invece, vengono sostituiti dal sintagma »l'uno ordine e l'altro«. Nella traduzione di Prepeluh non c'è uno sforzo simile volto ad aumentare la trasparenza delle relazioni del mondo testuale:

* Marco solo visse e morí onoratissimo, perché lui succedé allo imperio iure hereditario, e non aveva a riconoscere quello né da' soldati né da' populi; di poi, sendo accompagnato da molte virtú che lo facevano venerando, tenne sempre, mentre che visse, l'uno ordine e l'altro intra e' termini suoi, e non fu mai né odiato né disprezzato. (P 107)

* Samo Mark je živel in umrl v velikih časteh, ker je nastopil vlado iure hereditario in ni bilo treba, da bi bil zanjo hvaležen bodisi vojakom, bodisi ljudstvu. Spričo mnogih vrlin, zavoljo katerih so ga spoštovali, je dalje za živa zmeraj obdržal v pravih mejah oba sloja - ljudstvo in vojsko - in ga nikoli niso sovražili ne zaničevali. (K 70)

* Edino ta je živel in umrl češčen, ker je prišel do vladarstva po dednem pravu in mu zato ni bilo treba biti hvaležnemu niti vojaštvu niti ljudstvu. Razven tega so ga krasile mnoge čednosti; znal je ves čas svoje vlade pravilno zamejiti oba dela; nikoli ni bil niti osovražen niti zaničevan. (Prep 81-82)

\section{IV.2.2 Aggiunta di espressioni semanticamente vicine}

Legato alla tendenza ad aumentare la trasparenza del senso testuale è anche il procedimento consistente nell'integrazione di espressioni sinonimiche o semanticamente vicine, il che spesso crea dei sintagmi potenzialmente pleonastici. A mo' d'illustrazione può servirci il passo seguente, che contiene un brano del XIX capitolo del 
Principe, in cui viene discusso l'atteggiamento del popolo nei confronti del principe. Il termine »reverito《 viene reso da Košir con una combinazione di due termini (»spoštovati«/»rispettare«, »reverire $\ll /$ e »bati se«/»aver paura «/), grazie alla quale il testo d'arrivo si distacca notevolmente dal testo di partenza. Tuttavia la soluzione si rivela funzionale, poiché è in grado di rafforzare la coerenza testuale: corrisponde alla convinzione di Machiavelli che per un principe sia »molto più sicuro essere temuto che amato, quando si abbia a mancare dell'uno de' dua.« (Cap. XVII). Nella traduzione di Prepeluh l'espansione sinonimica non si trova:

(4)

* /.../ e contro chi è reputato con difficultà si coniura, con difficultà è assaltato, purché si intenda che sia eccellente e reverito da' suoi. (P 102)

* Proti tistemu pa, ki uživa čast, je težko kovati zarote, takega je težko napasti, le da se ve, da je izvrsten mož in da ga podložniki spoštujejo in se ga boje. (K 66) $* / \ldots /$ vsled tega je tudi zarota proti njemu težavna. Vsakdo se bo le težko odločil ga napasti, ker vé, da je mož velikih svojstev, češčen od svojcev. (Prep 78-79)

L'aggiunta di espressioni semanticamente vicine è caratteristica soprattutto della versione koširiana. La traduzione di Prepeluh, invece, tende piuttosto alla condensazione ovvero riduzione del messaggio all'essenziale, come appare dall'esempio (5), in cui i due procedimenti vanno visti come una riflessione delle diverse funzioni assegnate alle due traduzioni. Nell'esempio (5) possiamo osservare quanto Košir sia attento a non perdere i singoli elementi del messaggio, mentre Prepeluh preferisce una versione molto più laconica (letteralmente: »Poi deve un principe amare le virtù ed onorare gli uomini eccellenti in ogni arte.«):

(5)

* Debbe ancora uno principe mostrarsi amatore delle virtú dando recapito alli uomini virtuosi, e onorare gli eccellenti in una arte. (P 123)

* Vladar se mora dalje kazati kot človek, ki ceni sposobnosti, in sicer tako, da daje zavetje talentiranim ljudem in izkazuje čast tistim, ki se odlikujejo v kaki umetnosti. (K 82)

* Nadalje mora vladar ljubiti vrline in častiti izborne može vsake stroke. (Prep 92)

\section{IV.2.3 Introduzione di strutture parallele}

È generalmente noto che la presenza di vari tipi di strutture parallele - che possono essere considerate una specie di ripetizione - abbia un forte effetto connettivo nel testo (cfr. Hartnett 1986: 152, Charolles 1994: 131-132, Reinhart 1980: 169), per cui può contribuire alla trasparenza del senso. A parte ciò, tali strutture hanno anche una funzione retorico-stilistica. Ambedue le funzioni si fanno sentire nella traduzione di Košir, che usa i parallelismi più volte, mentre tale procedimento sembra assente nella 
traduzione di Prepeluh. Nell'esempio (6), abbiamo a che fare con la ripetizione della proposizione principale:

(6)

* E non è maraviglia se alcuno de' prenominati Italiani non ha possuto fare quello che si può sperare facci la illustre casa vostra; e se, in tante revoluzioni di Italia e in tanti maneggi di guerra, e' pare sempre che in quella la virtú militare sia spenta. (P 135)

* Ni čudno, če nobeden od prej omenjenih Italijanov ni mogel narediti tistega, kar bo, tako upamo, naredil vaš slavni rod. Prav tako ni čudno, če je po tolikih prekucijah in vojnih spletkah v Italiji še vedno videti, da so vojaške sposobnosti v njej zamrle. (K 93)

In ne smemo se čuditi, ako nobeden od imenovanih Italijanov ni mogel izvršiti ono, o čemer upamo, da bo izvršil vaš presvetli rod, in ako vspričo tolikih preobratov v Italiji in številnih vojn dozdevamo, da so ugasnile vse njene vojaške vrline. (Prep 101)

Questo esempio illustra allo stesso tempo anche la creazione di nuovi rapporti interfrastici nel testo d'arrivo rispetto al testo di partenza. Si tratta di un procedimento relativamente frequente, con il quale il processo di elaborazione cognitiva del testo viene semplificata. Vediamone un caso a parte.

\section{IV.2.4 Creazione di nuovi rapporti interfrastici}

Com'è noto, nella prosa antica i periodi tendono ad essere più lunghi che in testi moderni; i limiti proposizionali sono più sovente segnalati con la virgola, il punto e virgola o con i due punti a scapito del punto fermo (cfr. Serianni 1991: 70). Se nella traduzione in sloveno moderno tale punteggiatura si conservasse, i testi d'arrivo risulterebbero poco naturali ed eccessivamente complessi, per cui la loro elaborazione cognitiva potrebbe diventare assai ardua per il lettore. Per facilitare la costruzione della coerenza, i due traduttori, normalmente, creano delle unità più piccole. ${ }^{14}$ Eccone un esempio:

(7)

* Uno principe, pertanto, savio, sempre ha fuggito queste arme, e voltosi alle proprie; e ha volsuto piuttosto perdere con li sua che vincere con gli altri, iudicando non vera vittoria quella che con le armi aliene si acquistassi. Io non dubiterò mai di allegare Cesare Borgia e le sue azioni. Questo duca intrò in Romagna con le armi ausiliarie, conducendovi tutte gente franzesi; e con quelle prese Imola e Furlí; ma non li parendo poi tale arme secure, si volse alle mercenarie, iudicando in quelle

14 Lo stesso procedimento si può osservare anche in traduzioni slovene di altri testi italiani antichi, come, ad esempio, nella traduzione del Decameron fatta da Andrej Budal (1959) e soprattutto in quella di Niko Košir (1980). 
manco periculo; e soldò gli Orsini e Vitelli; le quali poi nel maneggiare trovando dubie ed infedeli e periculose, le spense, e volsesi alle proprie. (P 83-84)

* Vsak kolikor toliko moder vladar se je take vojske zmeraj izogibal in se zatekal k svoji. Rajši je celo s svojo izgubljal, kakor zmagoval s tujo, sodeč, da zmaga, ki bi si jo pridobil s tujim orožjem, ni prava. Nikoli si ne bom pomišljal navajati za zgled Cesara Borgio in njegova dejanja. Ta vojvoda je vdrl v Romagno s pomožno vojsko, saj je vodil s sabo same Francoze. $\mathbf{Z}$ njimi je zavzel Imolo in Forli. Ker pa se mu ta vojska ni zdela zanesljiva, se je kasneje zatekel k najemniški, sodeč, da je manj nevarna, pa je najel Orsine in Vitelle. Ko je po njeni rabi ugotovil, da je tudi ta nezanesljiva, nezvesta in nevarna, jo je odpravil in se zatekel $\mathrm{k}$ lastni. (K 51)

* Vsak vsaj nekoliko moder vladar se je takih čet vedno izogibal in je bil rajši premagan samostojen, kakor pa da bi zmagal s tujo pomočjo, dobro vedoč, da mu $\mathrm{s}$ tujo pomočjo priborjena zmaga ne bo $\mathrm{v}$ korist. Ne pomišljam staviti za zgled Cesare Borgia in njegova dejanja. On je s pomožnimi četami vdrl v Romagno; poveljeval je francoskemu vojaštvu, s katerim je zavzel Imolo in Furlí. Ker pa je spoznal njegovo nezanesljivost, se je odločil, da si nabere najemnikov, ki so se mu zdeli manj nevarni. Sprejel je v svojo službo Orsinije in Vitellije. Tekom njih vporabe je zapazil, da so tudi te čete nezanesljive, nezveste in nevarne. Zato jih je zopet razpustil in se ogledal po lastnih ljudeh. (Prep 65-66)

\section{IV.2.5 Uso di connettori testuali}

La funzione principale dei connettori testuali è quella di segnalare esplicitamente $\mathbf{i}$ rapporti del mondo testuale, rendendo così più facile l'elaborazione cognitiva del testo. Visto che Machiavelli stesso tende alla chiarezza e alla trasparenza delle relazioni logico-semantiche, il suo uso dei connettori è piuttosto frequente. A quanto pare, anche Košir si rendeva ben conto del ruolo di tali elementi, conservando quelli di Machiavelli e aggiungendone nuovi. Nella traduzione di Prepeluh, l'uso dei connettori non presenta alcuna sistematicità.

Ad es. nel caso seguente possiamo osservare l'introduzione del connettivo »namreč«, che ha valore esplicativo e risulta funzionale anche se non affatto indispensabile per l'interpretazione testuale:

(8)

* /.../ come fece ne' prossimi tempi papa Iulio; il quale, avendo visto nella impresa di Ferrara la trista prova delle sue armi mercenarie, si volse alle ausiliarie /.../ (P 82)

*/.../ tako je nedavno naredil papež Julij. Ko je namreč videl, kako klavrno se je v bojih okoli Ferrare izkazala njegova najemniška vojska, se je zatekel k pomožni l.../ (K 50)

*/.../ kakor je storil nedavno papež Julij. Po žalostnih skušnjah, ki jih je imel z najetimi četami pri Ferrari, se je pogodil /.../ (Prep 64) 


\section{$\checkmark$ Conclusione}

Come si è potuto vedere dall'analisi degli esempi, le due traduzioni slovene del Principe risultano assai diverse dal punto di vista della loro funzione nella cultura d'arrivo; entrambe sono, però, testi coerenti, in grado di avvicinare il mondo dell'opera machiavelliana al lettore sloveno. Ambedue le traduzioni presentano, rispetto al loro originale, delle modificazioni importanti che hanno una motivazione funzionale. Sarebbe forse, a questo punto, opportuno esaminare la tipologia dei procedimenti di coerenza più da vicino e cercare di capire il loro funzionamento nei testi tradotti in base $\mathrm{a}$ un corpus più esteso.

\section{Bibliografia}

MACHIAVELLI, Niccolò $\left({ }^{5} 1983\right)$ Il Principe. Cur. Ugo Dotti. Milano: Feltrinelli.

MACHIAVELLI, Niccolò (1920) Vladar. Trad. da Abditus [Albin Prepeluh]. Ljubljana: Zvezna tiskama.

MACHIAVELLI, Niccolò (1966) Vladar. Trad. da Niko Košir. Ljubljana: Mladinska knjiga. [Knjižnica Sivi kondor. XIV.]

MACHIAVELLI, Niccolò (1990) Vladar. In: idem, Politika in morala. Trad. da Niko Košir. Ljubljana: Slovenska matica, 5-95. [Filozofska knjižnica. XXXIII.]

II

de BEAUGRANDE, Robert-Alain/Wolfgang Ulrich DRESSLER (1992) Uvod v besediloslovje. Trad. da Aleksandra Derganc in Tjaša Miklič. Ljubljana: Park.

BLUM-KULKA, Shoshana (1986) »Shifts of Cohesion and Coherence in Translation.« In: Juliane House/ShoshanaBlum-Kulka (cur.), Discourse and Cognition in Translation and Second Language Acquisition Studies. Tübingen: Narr, 17-35.

BUBLITZ, Wolfram/Uta LENK/Eija VENTOLA (cur.) (1999) Coherence in Spoken and Written Discourse: How to Create It and How to Describe It. (Selected Papers from the International Workshop on Coherence, Augsburg, 24-27 April 1997.) Amsterdam/Philadelphia: Benjamins.

CHAROLLES, Michel (1994) »Cohésion, cohérence et pertinence du discours. «Travaux de Linguistique 29 , $125-151$.

HALLIDAY, M. A. K./Ruqaiya HASAN (1976) Cohesion in English. London/New York: Longman.

HARTNETT, Carolyn G. (1986) »Static and dynamic cohesion: signals of thinking in writing. « In: Barbara Couture (cur.), Functional Approaches to Writing Research Perspectives. London: Frances Pinter Publishers, 142-153

HELLMAN, Christina (1995) »The Notion of Coherence in Discourse. « In: Gert Rickheit/Christopher Habel (cur.), Focus and Coherence in Discourse Processing. Berlin/New York: Walter de Gruyter, 190-202.

HEYDRICH, Wolfgang/Fritz NEUBAUER/János S. PETÖFI/Emel SÖZER (cur.)(1989) Connexity and Coherence: Analysis of Text and Discourse. Berlin/New York: Walter de Gruyter.

MEUTSCH, Dietrich/Reinhold VIEHOFF (cur.) (1989), Comprehension of Literary Discourse: Results and Problems of Interdisciplinary Approaches. Berlin/New York: Walter de Gruyter.

MILOJEVIĆ SHEPPARD (1993) Morpho-Syntactic expansions in translation from English into Slovenian as a prototypical response to the complexity of the original. München: Otto Sagner.

OŽBOT, Martina (1997) Ustreznost sporočila v slovenskem prevodu »Zgodovine italijanske književnosti« A. Momigliana. (Magistrska naloga.) Ljubljana: Univerza v Ljubljani, Filozofska fakulteta, Oddelek za romanske jezike in književnosti.

OŽBOT, Martina (1999) Prevajalske strategije in vprašanje koherence ob slovenskem prevodu Machiavelijevih "zgodovinskopolitičnih spisov". (Doktorska disertacija.) Ljubljana: Univerza v Ljubljani, Filozofska fakulteta, Oddelek za romanske jezike in književnosti.

OŽBOT, Martina (ics) »Making sense across cultures: the establishment of coherence in translated texts. « In: Atti del Convegno della Società Britannica di Linguistica Applicata (Cambridge, 2000).

PETÖFI, János S./Terry OLIVI (1989) »Understanding literary texts: A semiotic textological approach.« In: D. Meutsch/R. Viehoff (cur.), 190-225. 
REINHART, Tanya (1980) »Conditions for Text Coherence.« Poetics Today I/4, 161-180.

REIß, Katharina/Hans J. VERMEER (1984) Grundlegung einer allgemeinen Translationstheorie. Tübingen: Niemeyer.

RESCH, Renate (1997) »Ein kohärentes Translat - was ist das? Die Kulturspezifik der Texterwartungen.« In: Mary Snell-Hornby/Zuzana Jettmarová/Klaus Kaindl (cur.), Translation as Intercultural Communication: Selected Papers from the EST Congress - Prague 1995. Amsterdam/Philadelphia: John Benjamins, 271-281.

SERIANNI, Luca (21991) Grammatica italiana: Italiano comune e lingua letteraria. Torino: UTET.

van de VELDE, Roger G. (1992) Text and Thinking: On Some Roles of Thinking in Text Interpretation. Berlin/New York: Walter de Gruyter.

VIEHWEGER, Dieter (1989) »Coherence - Interaction of Modules.« In: W. Heydrich/F. Neubauer/J. S. Petöfi/E. Sözer (cur.), 256-274.

VIPOND, Douglas/Russell A. HUNT (1989) »Literary Processing and Response as Transaction: Evidence for the Contribution of Readers, Texts, and Situations.« In: D. Meutsch/R. Viehoff (cur.), 155-174.

\section{Povzetek}

\section{BESEDILNA KOHERENCA S STALIŠČA PREVAJANJA}

Prispevek o vprašanju besedilne koherence $v$ prevodih prinaša izsledke raziskave, ki jo je spodbudilo opažanje, da prevodi v odnosu do izvirnikov pogosto izpričujejo posebno vrsto sprememb, pri katerih gre običajno za dodajanja elementov oz. skupin elementov. Te spremembe niso motivirane sistemsko, ampak funkcijsko, tj. z vlogo, ki naj bi jo dani prevod v ciljni kulturi opravljal. Glede na to je mogoče pričakovati, da bodo različni prevodi istega besedila vsebovali različne elemente, usmerjene h krepitvi besedilne koherence, $v$ odvisnosti od vsakokratne funkcije prevoda.

Po prikazu osnovnih značilnosti besedilne koherence in predstavitvi njenega mesta znotraj teorije skoposa, $v$ kateri zadobi koherenca posebno prevodoslovno relevantnost, se avtorica posveti slovenskim prevodom Machiavellijevega Vladarja. Izpostavi funkcijsko raznolikost Prepeluhove in Koširjeve verzije in skuša prepoznati postopke, s pomočjo katerih se vzpostavljanje koherence omogoča glede na predvideno funkcijo posameznega besedila. 